\title{
Partial loss of CovS function in Streptococcus pyogenes causes severe invasive disease
}

\author{
Ichiro Tatsuno, Ryo Okada, Yan Zhang, Masanori Isaka and Tadao Hasegawa*
}

\begin{abstract}
Background: CovRS (or CsrRS) is a two-component regulatory system that regulates the production of multiple virulence factors in Streptococcus pyogenes. covs mutations are often found in isolates recovered from mice that have been experimentally infected with $S$. pyogenes and covS mutations enhance bacterial virulence in an invasive infection mouse model. In addition, covS mutations were detected more frequently in a panel of clinical isolates from severe invasive streptococcal infections than those from non-severe infections. Thus, covs mutations may be associated with the onset of severe invasive infections.

Results: Known covS mutations were divided into two groups: (i) frameshift mutations that caused a deletion of functional regions and (ii) point mutations that caused single (or double) amino acid(s) substitutions. Frameshift mutations are frequent in mouse-passaged isolates, whereas point mutations are frequent in clinical isolates. The functions of CovS proteins with a single amino acid substitution in clinical isolates were estimated based on the streptococcal pyrogenic exotoxin B (SpeB) production and NAD+-glycohydrolase (NADase) activity, which are known to be regulated by the CovRS system. Point mutations partially, but not completely, impaired the function of the covs alleles. We also investigated some of the benefits that a partial loss of function in covs alleles with point mutations might confer on clinical isolates. We found that covS knockout mutants ( $\Delta$ covs strains) had an impaired growth ability in a normal atmosphere in Todd Hewitt broth compared with parental isolates having wild-type or point-mutated covs.

Conclusions: The loss of CovS proteins in S. pyogenes may confer greater virulence, but bacteria may also lose the ability to respond to certain external signals recognized by Covs. Therefore, point mutations that retain the function of CovS and confer hypervirulence may have natural selective advantages.
\end{abstract}

\section{Background}

Streptococcus pyogenes is a Gram-positive bacterium that infects the upper respiratory tract, including the tonsils and pharynx, which is responsible for post-infection diseases such as rheumatic fever and glomerulonephritis. S. pyogenes also causes severe invasive diseases including necrotizing fasciitis [1-5].

S. pyogenes is exclusively a human pathogen and it possesses many virulence factors that help it to resist host defense systems. The production of these factors is thought to be precisely regulated in response to host environmental conditions such as different infection sites or host immune system induction levels [6-8]. In

\footnotetext{
* Correspondence: tadaoh@med.nagoya-cu.ac.jp Department of Bacteriology, Nagoya City University Graduate School of Medical Sciences, 1 Kawasumi Mizuho-cho Mizuho-ku, Nagoya 467-8601, Japan
}

\section{Biomed Central}

prokaryotes, the regulation of protein production in response to fluctuating environmental conditions depends primarily on two-component regulatory systems, which consist of a sensor histidine kinase and its cognate response regulator [9]. Thirteen two-component regulatory systems have been described in S. pyogenes, of which the CovRS system (also known as the CsrRS system) mediates the control of several virulence factors [10-15]. Specific isolates from mice infected with $S$. pyogenes exhibited enhanced virulence in mice owing to spontaneous $\operatorname{cov} R$ or covS mutations $[10,15,16]$. In addition, covS mutations were detected more frequently in a panel of clinical isolates from severe invasive streptococcal infections than in a panel of clinical isolates from non-streptococcal toxic shock syndrome [10,16-18]. Thus, Ikebe et al. [18] suggested that $\operatorname{cov} S$ mutations are closely associated with the onset of streptococcal toxic shock syndrome. 
The strains used for experimental murine infections $[10,15,16]$ and clinical isolates [18] frequently have the M1 serotype, which is the most widely disseminated global serotype [19-21]. Engleberg et al. [15] showed that most $\operatorname{covS}$ mutations were frameshift or nonsense mutations in isolates from mice infected with the M1 strain. In contrast, all of the spontaneous changes in CovS detected in clinical M1 isolates [22] resulted from single amino acid substitutions. Thus, we were interested in why this difference occurred and we hypothesized that it was related to the use of animal-passaged isolates in the first study whereas the latter used clinical isolates. Several covS mutations have been reported in other studies $[10,16,18]$ in addition to the two mentioned previously $[15,22]$. In the current study, we first reviewed the different types of $\operatorname{cov} S$ mutations. This suggested that most of the spontaneous changes in CovS detected in clinical M1 isolates resulted from single amino acid substitutions, whereas most of the covS mutations detected in animal-passaged isolates were frameshift mutations. We also showed that covS mutations comprising single amino acid substitutions in the clinical isolates partially, but not completely, impaired the functions of CovS. Finally, we present some new findings and discuss why covS mutations in clinical isolates are preferentially single amino acid substitutions, whereas animal-passaged isolates tend to have frameshift mutations.

\section{Results and discussion}

\section{Classification of covs mutations}

We investigated all previously reported $\operatorname{cov} S$ mutations. The covS mutations were found most frequently in M1 isolates rather than any other serotypes $[10,15,18,22$, and our unpublished data]. The $\operatorname{cov} S$ mutations detected in M1 strains were divided into two groups: (i) frameshift or nonsense mutations that caused a deletion in functional regions and (ii) point mutations that caused single (or double) amino acid(s) substitutions. Of the $34 \operatorname{covS}$ mutations 25 were detected in isolates from mice infected with the M1 strain were frameshift mutations (Figure 1A and Additional file 1). In contrast, 16 of 29 $\operatorname{cov} S$ mutations detected in a panel of clinical isolates comprised single (or double) amino acid(s) substitutions (Figure $1 \mathrm{~B}$ and Additional file 2). Thus, significantly more frameshift mutations were detected in mousepassaged isolates, whereas point mutations were most frequent in clinical isolates $(P<0.05$, Fisher's exact test).

\section{Assessment of the function of CovS with an amino acid substitution using two-dimensional gel electrophoresis (2-DE)}

Theoretically, it is possible that a single amino acid substitution has no effect on CovS function, whereas a large deletion may affect domains that are critical for its function. However, we previously observed that all four clinical isolates (strains GT01, K2, AP04, and AP06) with covS alleles comprising single amino acid substitutions had lower SpeB production than a clinical isolate having the wild-type $\operatorname{cov} S$ when the culture supernatant proteins were analyzed by 2-DE [22]. It is known that $\operatorname{cov} S$ positively regulates $s p e B$ expression $[11,13,14]$, which suggests that mutated $\operatorname{cov} S$ alleles degrade the function of CovS [22]. Thus, we were interested in how the functions of CovS proteins with single amino acid substitutions were degraded in clinical isolates. Thus, we deleted the $\operatorname{cov} S_{G T 01}$ allele encoding CovS $_{\mathrm{GT} 01}$ A206S (a substitution of Ala206 with Ser) from the GT01 isolate. As shown in Figure 2, the resulting GT01 $\Delta$ covS had lower SpeB production than the parental GT01 isolate, suggesting that $\operatorname{CovS}_{\mathrm{GT} 01} \mathrm{~A} 206 \mathrm{~S}$ did not completely lose its function.

\section{Evaluation of the function of CovS with an amino acid substitution based on its NADase activity}

$S$. pyogenes secretes $\mathrm{NAD}^{+}$-glycohydrolase (NADase) as one of its virulence factors. According to a previous study [10], mouse-passaged derivatives of a strain carrying wild-type CovS exhibited high levels of NADase activity. A comparison of the entire genome of these strains also showed that one of the mouse-passaged derivatives had only one genetic change relative to the parental strain carrying the wild-type CovS, which consisted of a 7-bp insertion in covS. Therefore, we were interested in the NADase activity of clinical isolates with covS alleles containing single amino acid substitutions. Table 1 shows that strain $1529 \Delta \operatorname{cov} S$ had a NADase activity level of $93.5 \mathrm{U}$, which was higher than the level of 3.4 $\mathrm{U}$ in parental isolate 1529 with wild-type covS reported in our previous study [23]. In addition, the activity level of strain $1529 \Delta \operatorname{covS}(93.5 \mathrm{U})$ was higher than the levels of $62.9 \mathrm{U}, 57.0 \mathrm{U}, 59.8 \mathrm{U}, 60.5 \mathrm{U}$, and $59.4 \mathrm{U}$ found in the clinical isolates $\mathrm{K} 2, \mathrm{GT} 01, \mathrm{AP} 04, \mathrm{AP} 06$, and FI01, respectively, which had point-mutated covS alleles, in this study and a previous study [23], although isolate CR01 had a level of $114.3 \mathrm{U}$. The level of GT01 $\Delta$ covS was 105.0 U, which was higher than that of parental strain GT01 that carried CovS $_{\text {GT01 }}$ A206S. Thus, the NADase activity levels in isolates with point-mutated $\operatorname{covS}$ alleles were between those of isolates with wild-type covS and isolates with a complete deletion of $\operatorname{cov} S$. This was consistent with previous reports [22,23], where the levels of NADase in isolates KN01, MDYK, and MUY with wild type covS were $6.2 \mathrm{U}, 3.0 \mathrm{U}$, and $3.0 \mathrm{U}$, respectively.

Next, we attempted to complement $1529 \Delta \operatorname{cov} S$ with wild-type $\operatorname{cov} S_{1529}$ or derivatives, which were cloned into plasmid vector pLZ12-Km2. Wild-type $\operatorname{cov} S_{1529}$ from isolate 1529 was cloned into $\mathrm{pLZ}^{-\operatorname{covS}}{ }_{1529}$ and it reduced the NADase activity by $71.5 \mathrm{U}$ from $201.9 \mathrm{U}$ of 1529 $\Delta \operatorname{cov} S$ (pLZ12-Km2: control vector) to $130.4 \mathrm{U}$ of 


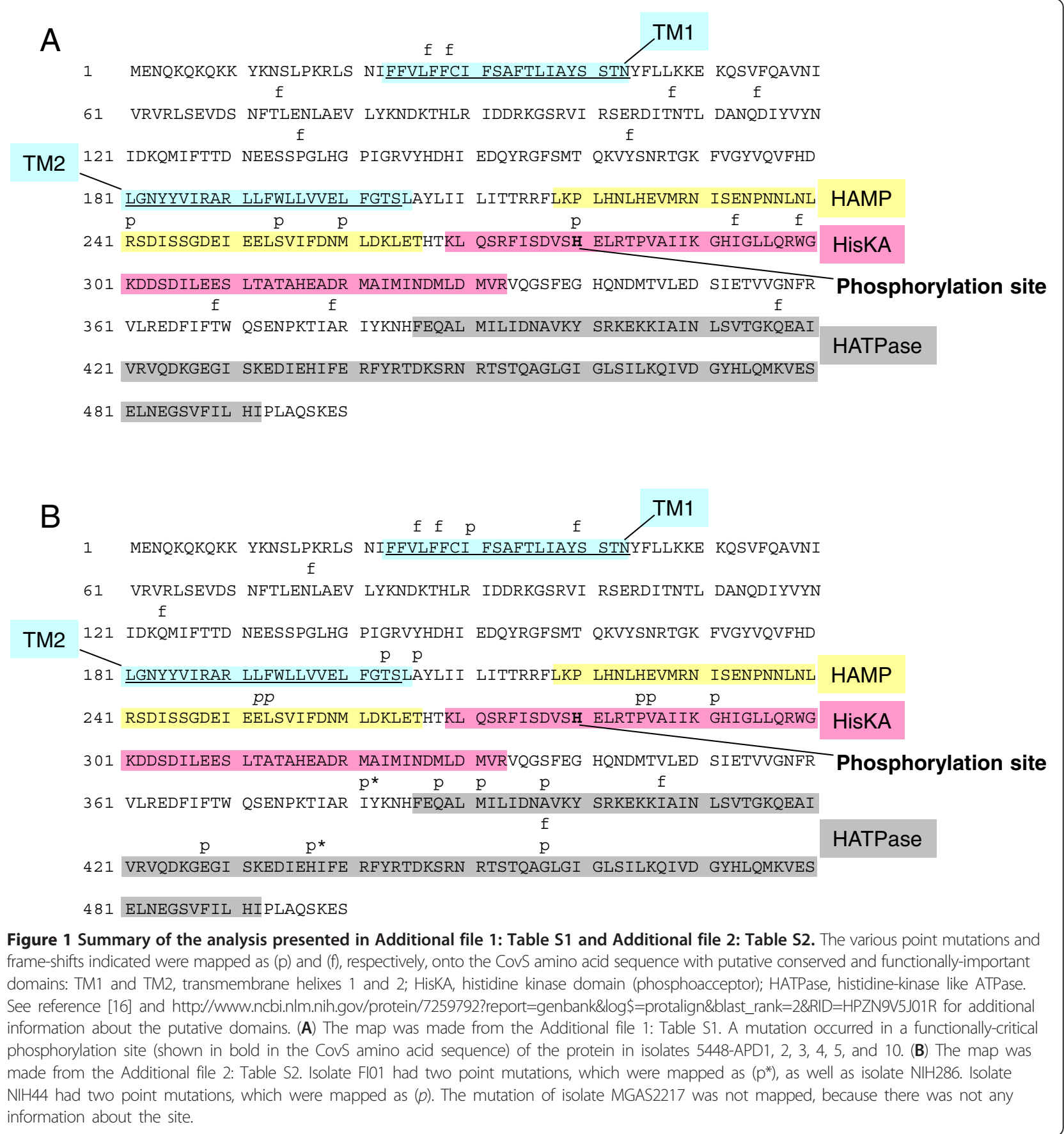

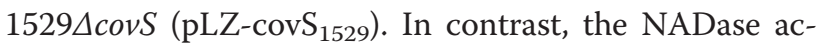
tivity levels in pLZ-covS ${ }_{1529} \mathrm{I} 30 \mathrm{~L}, \mathrm{pLZ}-\mathrm{covS}_{1529} \mathrm{E} 428 \mathrm{G}$, and pLZ-covS ${ }_{1529} \mathrm{~A} 206 \mathrm{~S}$ encoding mutated covS alleles from isolates K2, AP04 (or AP06), and GT01 were reduced by $39.2 \mathrm{U}, 17.1 \mathrm{U}$, and $15.2 \mathrm{U}$, respectively (Table 1 and Figure 2). Thus, the pLZ-covS ${ }_{1529} \mathrm{I} 30 \mathrm{~L}$, the pLZ$\operatorname{covS}_{1529}$ E428G, and the pLZ-covS ${ }_{1529} \mathrm{~A} 206 \mathrm{~S}$ certainly retained their abilities to reduce NADase activity, but the abilities were lower than that of the $\mathrm{pLZ}^{-\operatorname{cov} S_{1529}}$ with wild-type covS.
These results suggest that amino acid substitutions, such as I30L, E428G, and A206, partially impaired the function of CovS.

\section{Benefits of partially impaired CovS}

According to previous studies [10,16-18], CovS negatively regulates the expression of certain virulent genes; therefore, a mutation in $\operatorname{cov} S$ may increase the virulence in mouse models of infection where it plays a crucial role in the onset of severe invasive infections. However, 


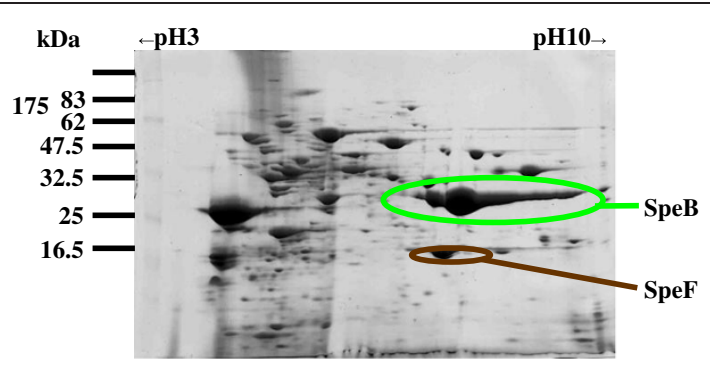

A (GT01 wild)

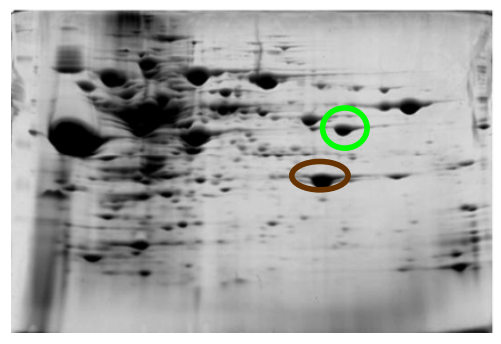

B (GT01 $\Delta$ covS)

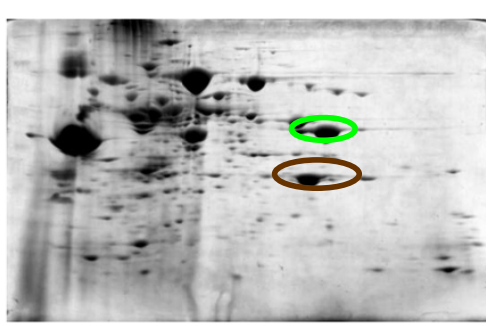

$\mathrm{C}(\mathrm{SF} 370 \Delta \operatorname{cov} S)$

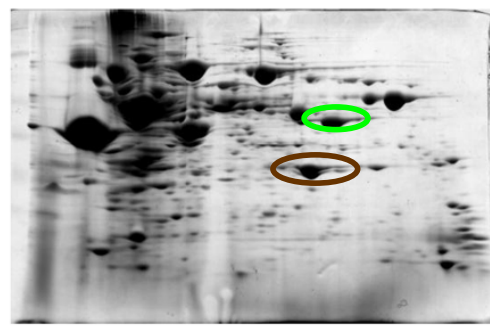

$\mathrm{D}(1529 \Delta \operatorname{covS})$

Figure 2 Two-dimensional gel electrophoresis (2-DE) analysis of exoproteins from S. pyogenes GT01 (A), GT014covS (B), SF3704covS (C), and 15294covS (D). S. pyogenes strains were cultured in $25 \mathrm{ml} \mathrm{BHI-Y} \mathrm{broth} \mathrm{and} \mathrm{the} \mathrm{proteins} \mathrm{in} \mathrm{the} \mathrm{culture} \mathrm{supernatants} \mathrm{were} \mathrm{subjected} \mathrm{to}$ 2-DE analysis using $13 \mathrm{~cm}$ Immobiline Drystrip gels (pH 3-10; GE Healthcare Biosciences Co.). Some protein spots identified by LC-MS/MS analysis are shown. The SpeB spot sizes produced from GT014covS (B), SF3704covS (C), and 15294covS (D) were smaller than those from GT01 (A).

Table 1 NADase activity of S. pyogenes strains

\begin{tabular}{|c|c|c|c|c|}
\hline Strain & covs & $\operatorname{cov} R$ & NADase $\left(U^{\mathrm{a}}\right)$ & Reference \\
\hline 1529 & wt & wt & $3.4 \pm 0.7$ & [23] \\
\hline 15294 covs & $\Delta$ covs & wt & $93.5 \pm 3.5$ & this study \\
\hline $\mathrm{K} 2$ & I30L & wt & $62.9 \pm 4.6$ & this study \\
\hline GT01 & A206S & wt & $57.0 \pm 3.6$ & [23] \\
\hline CR01 & M391R & wt & $114.3 \pm 8.7$ & [23] \\
\hline AP04 & E428G & wt & $59.8 \pm 2.6$ & this study \\
\hline AP06 & E428G & wt & $60.5 \pm 5.4$ & this study \\
\hline $\mathrm{Fl01}$ & $1381 \mathrm{~T}+\mathrm{H} 437 \mathrm{R}$ & wt & $59.4 \pm 4.8$ & [23] \\
\hline $1529 \Delta \operatorname{cov} R$ & wt & $\Delta \operatorname{cov} R$ & $106.7 \pm 3.7$ & this study \\
\hline GT014covs & $\Delta$ covs & wt & $105.0 \pm 3.2$ & this study \\
\hline GT01 1 covR & wt & $\Delta \operatorname{cov} R$ & $103.5 \pm 6.7$ & this study \\
\hline $1529 \Delta$ covs (pLZ12-km2) & $\Delta$ covs & wt & $201.9 \pm 2.8$ & this study \\
\hline $1529 \Delta \operatorname{covS}\left(\mathrm{pLZ}-\operatorname{covS}_{1529}\right)$ & wt & wt & $130.4 \pm 3.4$ & this study \\
\hline $1529 \Delta$ covS (pLZ-covS $\left.{ }_{1529} \mid 381 L\right)$ & I381T & wt & $176.7 \pm 8.9$ & this study \\
\hline 15294covS (pLZ-covS ${ }_{1529}$ H437R) & H437R & wt & $114.5 \pm 6.8$ & this study \\
\hline $15294 \operatorname{covs}\left(\mathrm{pLZ}-\operatorname{covS}_{1529} 130 \mathrm{~L}\right)$ & $\mathrm{I} 30 \mathrm{~L}$ & wt & $162.7 \pm 11.0$ & this study \\
\hline 1529AcovS (pLZ-covS ${ }_{1529}$ E428G) & E428G & wt & $184.8 \pm 6.9$ & this study \\
\hline 15294 covs (pLZ-covS ${ }_{1529}$ A206S) & A206S & wt & $186.7 \pm 4.2$ & this study \\
\hline 1529 (pLZ12-km2) & wt & wt & $2.4 \pm 0.16$ & this study \\
\hline
\end{tabular}

${ }^{a}$ NADase activity (Units) \pm standard error are indicated. One unit of NADase activity is defined as the amount $(\mu \mathrm{g})$ of $\beta$-NAD cleaved per hour per $\mu$ c culture supernatant, as described previously $[23,31]$. 
the loss of CovS function means that S. pyogenes can no longer adjust to environmental fluctuations. For example, environmental $\mathrm{Mg}^{2+}$ is thought to be recognized by the CovS sensor protein [24]. Therefore, the partial loss of CovS function may be favorable in nature, but not under laboratory conditions. This hypothesis led us to investigate the benefits of $\operatorname{cov} S$ in S. pyogenes. Previously, Trevino et al. [25] showed that a $\operatorname{cov} S$ mutated strain had a lower growth ability than the parental wildtype strain in human saliva, but not in Todd Hewitt broth, which is the standard broth used to culture $S$. pyogenes. We were interested in the factor present in human saliva that is recognized by CovS; therefore, we repeated this experiment using the isolates 1529, SF370, and GT01. Bacteria were cultured under essentially the same conditions as those described previously [25]. However, the CFU (colony forming units)/ml for overnight THY broth cultures of strains 1529 $\Delta$ covS, SF370 $\Delta \operatorname{cov} S$, and GT01 $\Delta \operatorname{cov} S$ were lower than or similar to those of their parental strains; i.e., 1529, SF370, and GT01, respectively (Figure 3A), whereas the CFU/ml for overnight THY broth cultures of the isogenic mutant 2221covS::7 bp was four times that of the parental strain MGAS2221 reported in the previous study [25]. Thus, we observed two discrepancies: (i) between our results using isolates 1529, SF370, and GT01 (Figure 3A), and the previous results based on isolate MGAS2221 [25] and (ii) between our results with isolate 1529 (or GT01) and SF370. These discrepancies may be because of strain specificities. We did not have strain MGAS2221, so we further investigated the discrepancy between strains SF370 and GT01 or 1529. First, we analyzed the growth curves of the covS mutated strains. SF370 $\Delta$ covS, 1529 $\Delta$ covS, and GT01 $\Delta$ covS all showed delayed growth compared with that of their parental strains; i.e., SF370, 1529, and GT01, respectively (Figures $4 \mathrm{~A}-\mathrm{C}$ ). Thus, there was no discrepancy between strains SF370, GT01, and 1529 in terms of their growth kinetics. In addition, GT01 exhibited delayed and advanced growth compared with strain 1529 (or SF370) and strain GT01 $\Delta$ covs (Figures 4D and C), which was consistent with our hypothesis that the A206S substitutions partially impaired the function of CovS.

The growth abilities of SF370 and its isogenic covS mutant SF370 4 covS in THY broth differed from each other when evaluated on the basis of, but not the CFU/ml in overnight cultures (Figure 3A), the growth curves (Figure 4A). This new discrepancy may have occurred because the overnight culture, but not the growth curve, was conducted in $5 \% \mathrm{CO}_{2}$, which was the condition described in a previous study [25]. Therefore, we prepared overnight cultures of wild-type SF370 (SF370wt) and SF370 $\Delta$ covS in natural atmosphere (NA) conditions. As shown in Figure $3 \mathrm{~B}$, the $\mathrm{CFU} / \mathrm{ml}$ for SF370 $\Delta \operatorname{cov} S$ was lower than that of its parental strain SF370. Finally, we performed supplementary and supporting experiments to test the reliability of this study. $\operatorname{cov} S$ and $\operatorname{cov} R S$ cloned into a plasmid vector complemented the delayed growth of $1529 \Delta$ covS (Figure 5). pLZ-covS ${ }_{1529}$ and pLZ-covRS ${ }_{1529}$ increased the $\mathrm{CFU} / \mathrm{ml}$ for overnight THY broth cultures of strain 1529 $\Delta \operatorname{cov} S$ (Figure 6). As shown in Figure 7, $1529 \Delta$ covS was hypervirulent in a mouse infection model compared with the parental strain $1529(\mathrm{P}<0.01)$, as shown with other strains and their isogenic $\Delta$ covS mutants in previous studies $[10,16]$.

\section{Conclusions}

On the basis of our results and those from previous studies we concluded that the loss of $\operatorname{cov} S$ increases the

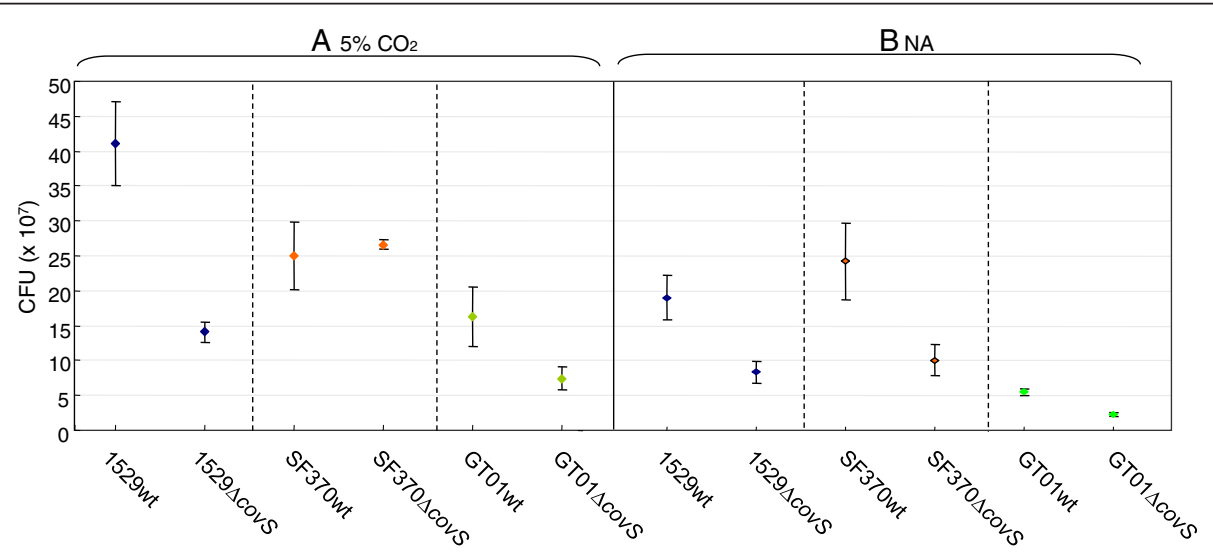

Figure 3 Growth ability of covs mutant S. pyogenes in THY broth. The CFU/ml after $23 \mathrm{~h}$, THY broth culture of isogenic covs mutant strains (1529 4 covS, SF370 4 covS, and GT01 AcovS), and parental isolates (1529, SF370, and GT01) are shown. At least three independent experiments were performed and they always yielded essentially the same results. The error bars indicate the standard errors of the means. (A) 5\% CO2 was used as an experimental condition. The CFU/ml for overnight THY broth cultures of strains 1529AcovS and GT014covS were lower than those for their parental strains 1529 and GT01, respectively. The CFU/ml for overnight THY broth cultures of SF3704covS were similar to that for the parental strain SF370. (B) Natural atmosphere (NA) was used as an experimental condition. 


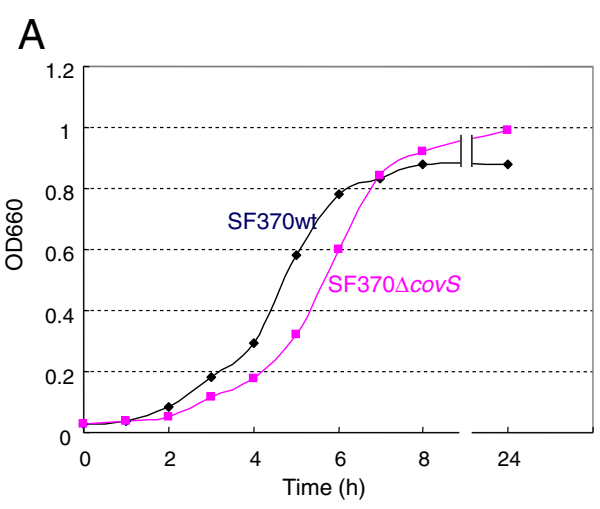

B

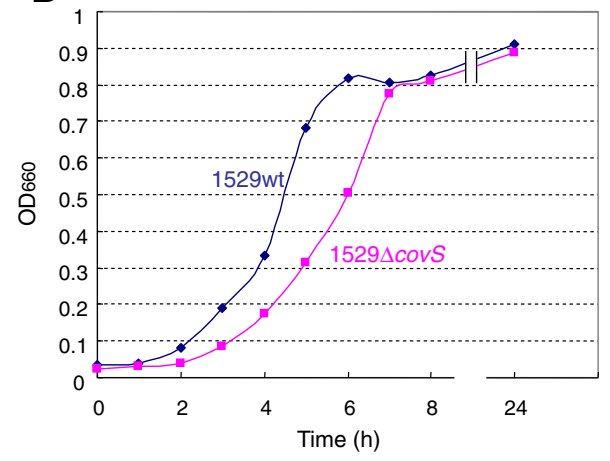

C

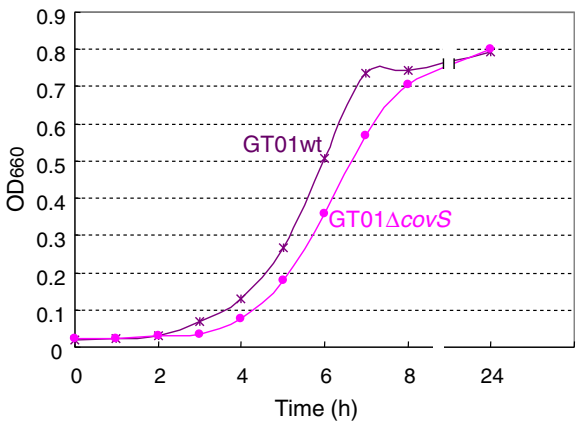

$\mathrm{D}$

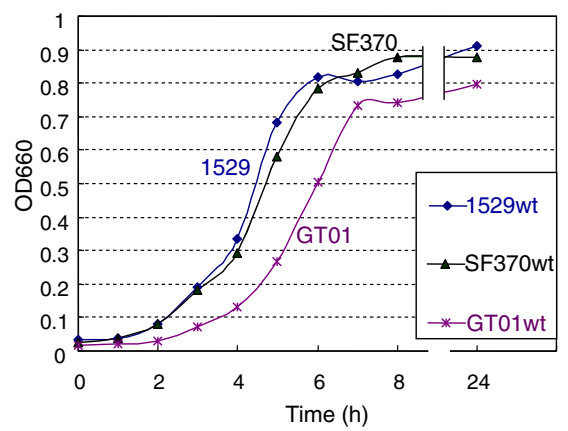

Figure 4 Growth curves of covS null mutants in THY broth. Three independent experiments produced essentially the same results. Representative data from three independent experiments are shown. (A-C) SF3704covS, 15294covS, and GT014covS all exhibited delayed growth compared with that of their parental strains SF370, 1529, and GT01, respectively. (D) GT01 exhibited delayed growth compared with strains 1529 and SF370.

virulence of $S$. pyogenes (which is advantageous in vivo). However, the loss of $\operatorname{cov} S$ also impaired the growth ability of this organism in THY broth (which is disadvantageous in vitro). Therefore, the CovRS system may confer benefits in stages when virulent gene expression is not required. The expression of many genes is precisely regulated so they are expressed only when required; e.g.,

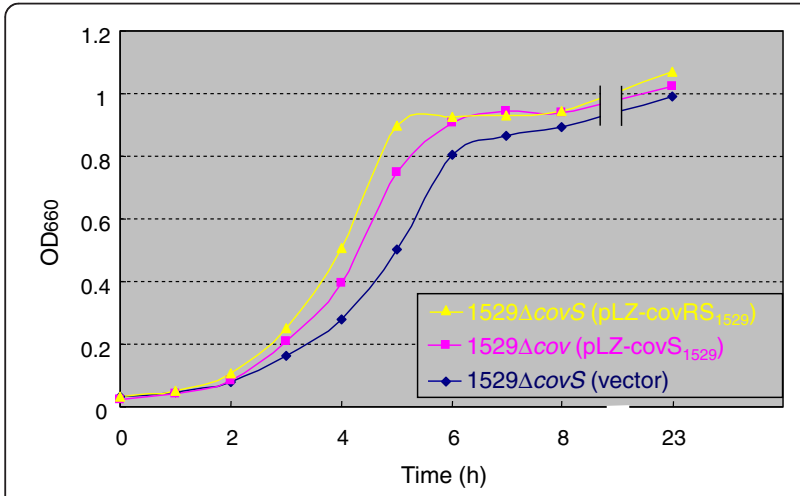

Figure 5 Growth curves of streptococcal strains in THY broth. Bacteria were cultured in THY broth supplemented with kanamycin $(62.5 \mathrm{\mu g} / \mathrm{ml})$ and the experiments were performed as described in Figure 4. catabolite repression. Therefore, partial attenuation of the CovRS system to promote resistance to the host defense system appears to be a wise choice for survival in nature.

We did not determine the components recognized by the CovS sensor proteins in our experimental conditions; i.e., THY broth, natural atmosphere, or $5 \% \mathrm{CO}_{2}$. However, it was probably not the $\mathrm{Mg}^{2+}$ ion, which was suggested previously [24], because we did not add $\mathrm{Mg}^{2+}$ ion to THY broth. Therefore, we propose that CovS can sense other signals in addition to the $\mathrm{Mg}^{2+}$ ion.

\section{Methods}

\section{Bacterial strains}

Streptococcal strains were isolated as the causative organisms in patients from Japan [22,23]. S. pyogenes (GAS) strain SF370, which was the most prevalent database reference isolate (accession number NC_002737), was provided by J. J. Ferretti [26,27]. Streptococcal strains were cultured in brain-heart infusion (E-MC62, EIKEN Chemical Co., Tokyo, Japan) supplemented with $0.3 \%$ yeast extract (BD, Sparks, MD, USA), (BHI-Y) broth or Todd Hewitt broth (BD, Sparks, MD, USA) supplemented with $0.2 \%$ yeast extract broth (THY) unless otherwise stated. 


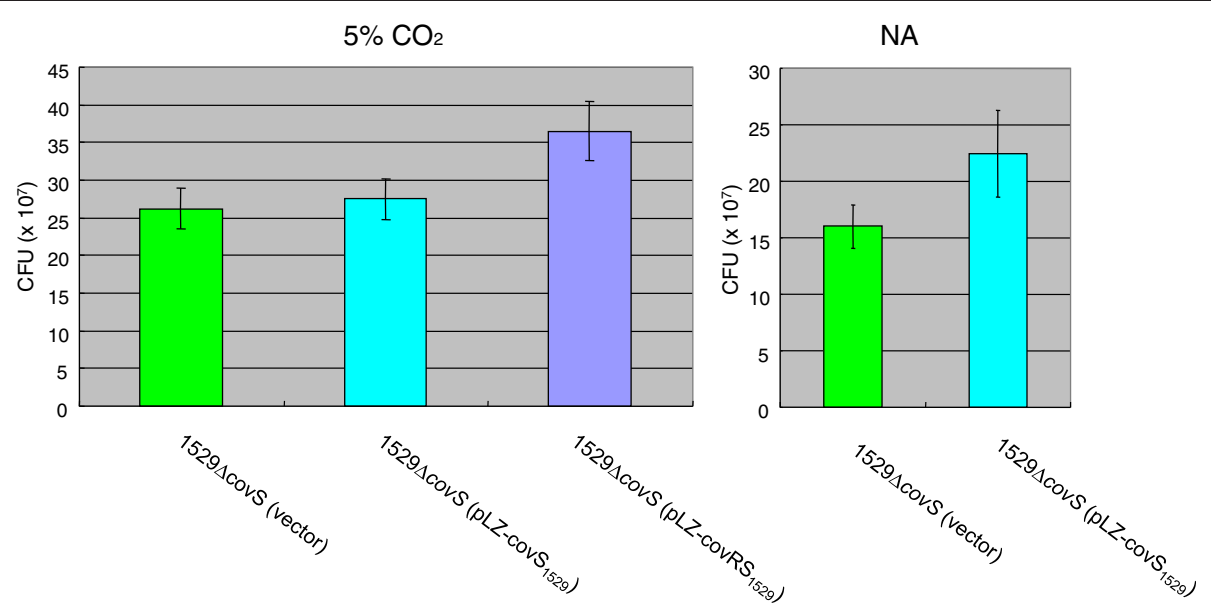

Figure 6 Growth of covS mutant S. pyogenes in THY broth with $5 \% \mathrm{CO}_{2}$ or NA. These experiments were performed as described in Figures 3. The error bars indicate the standard errors of the means.

\section{Production of covs knockout strains}

We constructed S. pyogenes strain $1529 \Delta \operatorname{cov} S$ as described previously [28]. Strains GT01 $\Delta \operatorname{cov} S$ and SF370 $\Delta \operatorname{cov} S$ were constructed using the same strategy [28].

\section{Two-dimensional gel electrophoresis (2-DE)}

Each bacterial isolate was cultured in BHI-Y at $37^{\circ} \mathrm{C}$ overnight without agitation. Exoproteins from the culture supernatant were prepared as described previously [22]. In brief, all sample pellets derived from bacterial culture supernatant were dissolved in dehydration solution, which consisted of $7.8 \mathrm{M}$ urea, $2 \mathrm{M}$ thiourea, $2 \%$ CHAPS, $0.6 \%$ dithiothreitol, and 0.5\% IPG buffer. The samples were loaded onto $13 \mathrm{~cm}$ Immobiline DryStrip gels (pH 3-10, GE Healthcare Biosciences Co. Piscataway, NJ, USA). The first-dimensional electrophoresis conditions were carried out according to the manufacture's instruction. Second-dimensional SDS-PAGE separation was performed as described previously [22]. The experiments were repeated at least 3 times to confirm their reproducibility.

\section{Production of covR knockout strains}

To construct the plasmid for the $\operatorname{cov} R$ knockout mutant, the $5^{\prime}$ end of $\operatorname{cov} R$ (fragment 1 ) was amplified using the oligonucleotide primer covR-n6 (5'-GGCTAGCCTTT AGAGAATATGGTTACT-3') with an NheI restriction site and primer covR-c2 (5'-TCCCCCGGGCTTTGTCA TTTATACCAACC-3') with an SmaI restriction site, while the $3^{\prime}$ end of $\operatorname{covR}$ (fragment 2) was amplified using the primer covR-n7 (5'-TCCCCCGGGGAGAAATAAGTC ATATGGAA-3') with an SmaI restriction site and primer

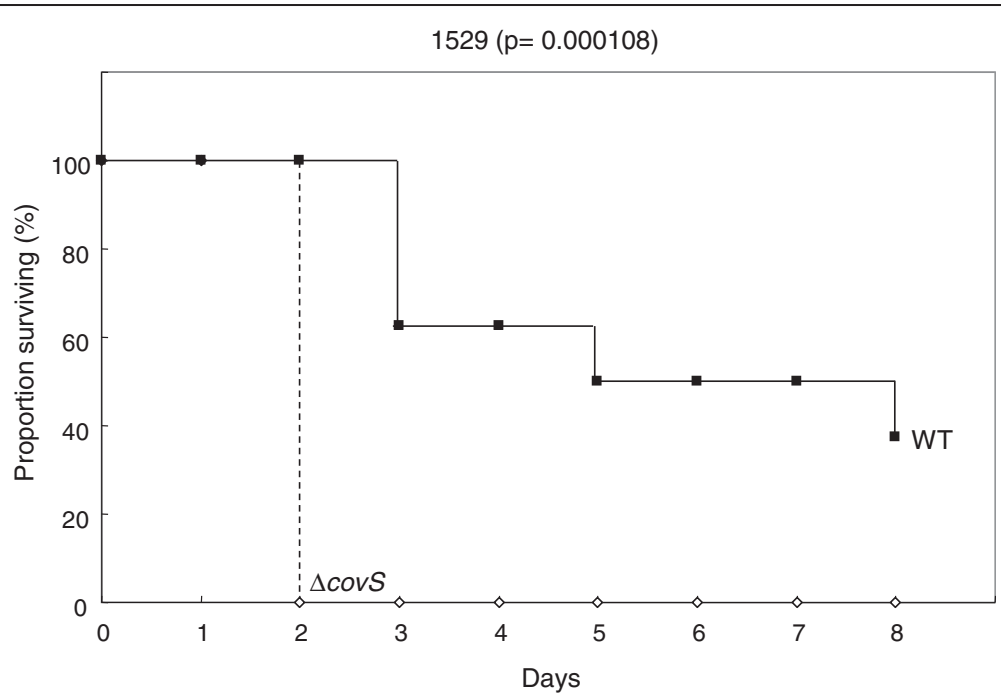

Figure 7 Survival after skin inoculation with S. pyogenes strain 1529 or isogenic mutant 15294 covS. Data were from two independent experiments and a total of eight mice for each challenged strain. $P<0.01$ for comparison between strains. 
covS-c10 (5'-GGACTAGTATGTAAAATTAGAGTCCAC C-3') with an SpeI restriction site. Fragment 2 was digested with SmaI and SpeI before its insertion into multicloning site 2 in the plasmid pFW12 [29]. The resulting plasmid was digested using NheI and $S m a \mathrm{I}$, and the spc1 DNA fragment containing aad9 (promoterless spectinomycin resistance gene), which was obtained from a SmaI-digested fragment of pSL60-1 [29], and the NheISmaI-digested fragment 1 were inserted. This plasmid,

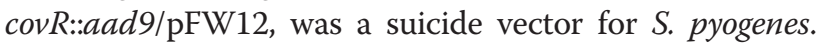
To prepare competent cells, strains 1529 and GT01 were harvested in the early to mid-log phase $\left(\mathrm{OD}_{660}, 0.4\right)$ and washed twice with $0.5 \mathrm{M}$ sucrose buffer. The suicide vector construct, covR::aad9/pFW12, was transformed into strains 1529 and GT01 via electroporation. The conditions for electroporation were $1.25 \mathrm{kV} / \mathrm{mm}, 25-\mu \mathrm{F}$ capacitance, and $200-\Omega$ resistance, and it was performed using a GenePulser II instrument (Bio-Rad, Hercules, CA). After incubation at $37^{\circ} \mathrm{C}$ for $3 \mathrm{~h}$, competent cells were spread onto BHI agar plates containing $0.3 \%$ yeast extract and spectinomycin (final concentration, $100 \mu \mathrm{g} / \mathrm{ml}$ ). Selected colonies were cultured from the plates. The cultured bacteria were washed once with saline, resuspended in $10 \mathrm{mM}$ Tris-1 mM EDTA, and boiled for $10 \mathrm{~min}$. Genomic DNA was obtained from the supernatant of the boiled bacteria. The double-crossover replacement was analyzed by PCR using genomic DNA. Successful doublecrossover replacement was further confirmed by DNA sequencing.

\section{Quantification of the NADase activity in the bacterial supernatant}

NADase activity was determined using the method of Stevens et al. [30] as described previously [31].

\section{Plasmids}

pLZ-covS $_{1529}$, pLZ-covS ${ }_{1529} \mathrm{I} 30 \mathrm{~L}$, and $\mathrm{pLZ-covS_{1529 }} \mathrm{E}_{428 \mathrm{G}}$ were constructed as described previously [22]. To construct pLZ-covS ${ }_{1529} \mathrm{~A} 206 \mathrm{~S}$, the DNA fragment was amplified using the oligonucleotide primers covR-n2 (5'-CTTTAGAGAATATGGTTACT-3'), covS-c2 (5'-GT AATTACATTTTGGACAAC-3'), and GT01 genomic DNA as templates with TaKaRa Ex Taq DNA polymerase (Takara, Ohtsu, Japan). The fragment consisted of $\operatorname{cov} R_{G T O 1}, \operatorname{cov} S_{G T O 1}$, and their $5^{\prime}$-noncoding region, which possibly contained the promoter region. This fragment was cloned into the pGEM-T vector (Promega, Madison, WI, USA). The resultant plasmid was digested with EcoRI and ligated into the same site in the pLZ12-Km2 plasmid [32] (pLZ-covRS $\mathrm{GTO1}_{\mathrm{GT}}$ ). To construct a plasmid containing only the $\operatorname{cov} S_{G T O 1}$ region, inverse PCR was conducted using two primers, covR-c2Sma (5'-TCCCCCGGGC TTTGTCATTTATACCAACC-3') and covR-n7Sma (5'TCCCCCGGGGAGAAATAAGTCATATGGAA-3'), with
pLZ-covRS $_{\text {GT01 }}$ plasmid DNA as template and PrimeSTAR HS DNA polymerase (Takara) to eliminate the $\operatorname{cov} R$ region. This blunt-ended PCR product was treated with $\mathrm{T} 4$ polynucleotide kinase (Takara) and self-ligated. The resultant plasmid was pLZ-covS ${ }_{1529}$ A206S. pLZ-covRS ${ }_{1529}$ encoding the $\operatorname{cov} R S_{1529}$ operon of isolate 1529 was constructed as described previously [22]. All of the $\operatorname{cov} R S$ DNA sequences were confirmed by sequencing.

\section{Mouse model of invasive skin tissue infection}

All animal studies conducted comply with federal and institutional (the Committee on the Ethics of Animal Experiments of the Nagoya City University) guidelines. The protocol was approved by the Committee on the Ethics of Animal Experiments of the Nagoya City University (Permit Number: H23M-07). All efforts were made to minimize suffering.

The ability of S. pyogenes to cause local skin lesions and necrosis in mice after skin inoculation was assessed using a similar procedure to that described previously [23,33]. Three-week-old female ICR mice (10-12 g) were anesthetized with sevoflurane and the skin of the left flank was laid bare by separating the hair with an alcohol swab, unless indicated otherwise. Bacteria $(0.2 \mathrm{ml} ; 2 \times$ $10^{7} \mathrm{CFU} /$ mouse) grown in BHI-Y were injected immediately beneath the surface of the skin using a 27-gauge needle so a superficial bleb appeared below the skin surface. The number of CFU injected was verified in each experiment by plating bacteria on BHI-Y or sheep blood agar plates and counting the CFU.

\section{Statistical analysis}

The survival times were assessed using a log-rank comparison. The $\mathrm{R}$ program was used for the statistical analysis http://bioinf.wehi.edu.au/software/russell/logrank/ webcite. $P \leq 0.05$ was considered significant.

\section{Availability of supporting data}

There are two supplementary tables.

\section{Additional files}

Additional file 1: Table S1. csrS mutations from mouse-passaged isolates of M1 S. pyogenes.

Additional file 2: Table S2. csrS mutations from human clinical isolates of M1 S. pyogenes.

\section{Abbreviations}

NADase: NAD ${ }^{+}$-glycohydrolase; NA: Natural atmosphere; THY: Todd Hewitt yeast.

\section{Competing interests}

There are no competing interests. 


\section{Authors' contributions}

IT conceived the study. IT, RO, and TH designed and performed the experimental work with help by $\mathrm{YZ}$ and MI. All authors contributed to the data analysis. IT wrote the original manuscript. TH helped to produce the final manuscript. All authors approved the final manuscript.

\section{Acknowledgments}

We thank Hideyuki Matsui for technical assistance, and Drs. M. Ohnishi, M. Ato, T. Ikebe for their helpful advices. This study was supported by JSPS KAKENHI Grant number 21790425 and 24590531, a grant from Ohyama Health Foundation, and a grant from the $24^{\text {th }}$ General Assembly of the Japanese Association of Medical Sciences (Medical Science Promotion Fund). The authors would like to thank Enago (www.enago.jp) for the English language review.

Received: 7 January 2013 Accepted: 20 March 2013

Published: 28 March 2013

\section{References}

1. Cone LA, Woodard DR, Schlievert PM, Tomory GS: Clinical and bacteriologic observations of a toxic shock-like syndrome due to Streptococcus pyogenes. N Engl J Med 1987, 317:146-149.

2. Hoge CW, Schwartz B, Talkington DF, Breiman RF, MacNeill EM, Englender SJ: The changing epidemiology of invasive group A streptococcal infections and the emergence of streptococcal toxic shock-like syndrome. A retrospective population-based study. JAMA 1993, 269:384-389.

3. Schwartz B, Facklam RR, Breiman RF: Changing epidemiology of group A streptococcal infection in the USA. Lancet 1990, 336:1167-1171.

4. Stevens DL: Invasive group A streptococcal infections: the past, present and future. Pediatr Infect Dis J 1994, 13:561-566.

5. Hasegawa T, Hashikawa SN, Nakamura T, Torii K, Ohta M: Factors determining prognosis in streptococcal toxic shock-like syndrome: results of a nationwide investigation in Japan. Microbes Infect 2004, 6:1073-1077.

6. Agarwal S, Agarwal S, Pancholi P, Pancholi V: Role of serine/threonine phosphatase (SP-STP) in Streptococcus pyogenes physiology and virulence. J Biol Chem 2011, 286:41368-41380.

7. Musser JM, Shelburne SA 3rd: A decade of molecular pathogenomic analysis of group A Streptococcus. J Clin Invest 2009, 119:2455-2463.

8. Kreikemeyer $B$, Mclver KS, Podbielski A: Virulence factor regulation and regulatory networks in Streptococcus pyogenes and their impact on pathogen-host interactions. Trends Microbiol 2003, 11:224-232.

9. Stock AM, Robinson VL, Goudreau PN: Two-component signal transduction. Annu Rev Biochem 2000, 69:183-215.

10. Sumby P, Whitney AR, Graviss EA, DeLeo FR, Musser JM: Genome-wide analysis of group $A$ streptococci reveals a mutation that modulates global phenotype and disease specificity. PLoS Pathog 2006, 2:e5.

11. Levin JC, Wessels MR: Identification of $c s r R / c s r S$, a genetic locus that regulates hyaluronic acid capsule synthesis in group A Streptococcus. Mol Microbiol 1998, 30:209-219.

12. Bernish B, van de Rijn I: Characterization of a two-component system in Streptococcus pyogenes which is involved in regulation of hyaluronic acid production. J Biol Chem 1999, 274:4786-4793.

13. Federle MJ, Mclver KS, Scott JR: A response regulator that represses transcription of several virulence operons in the group A streptococcus. J Bacteriol 1999, 181:3649-3657.

14. Heath A, DiRita VJ, Barg NL, Engleberg NC: A two-component regulatory system, CsrR-CsrS, represses expression of three Streptococcus pyogenes virulence factors, hyaluronic acid capsule, streptolysin $\mathrm{S}$, and pyrogenic exotoxin B. Infect Immun 1999, 67:5298-5305.

15. Engleberg NC, Heath A, Miller A, Rivera C, DiRita VJ: Spontaneous mutations in the CsrRS two-component regulatory system of Streptococcus pyogenes result in enhanced virulence in a murine model of skin and soft tissue infection. J Infect Dis 2001, 183:1043-1054.

16. Walker MJ, Hollands A, Sanderson-Smith ML, Cole JN, Kirk JK, Henningham A, McArthur JD, Dinkla K, Aziz RK, Kansal RG, Simpson AJ, Buchanan JT, Chhatwal GS, Kotb M, Nizet V: DNase Sda1 provides selection pressure for a switch to invasive group A streptococcal infection. Nat Med 2007, 13:981-985
17. Ato M, Ikebe $\mathrm{T}$, Kawabata $\mathrm{H}$, Takemori $\mathrm{T}$, Watanabe $\mathrm{H}$ : Incompetence of neutrophils to invasive group A streptococcus is attributed to induction of plural virulence factors by dysfunction of a regulator. PLOS One 2008, 3:e3455.

18. Ikebe T, Ato M, Matsumura T, Hasegawa H, Sata T, Kobayashi K, Watanabe H: Highly frequent mutations in negative regulators of multiple virulence genes in group A streptococcal toxic shock syndrome isolates. PLOS Pathog 2010, 6:e1000832

19. Rogers S, Commons R, Danchin MH, Selvaraj G, Kelpie L, Curtis N, Robins Browne $R$, Carapetis JR: Strain prevalence, rather than innate virulence potential, is the major factor responsible for an increase in serious group A streptococcus infections. J Infect Dis 2007, 195:1625-1633.

20. Aziz RK, Pabst MJ, Jeng A, Kansal R, Low DE, Nizet V, Kotb M: Invasive M1T1 group A Streptococcus undergoes a phase-shift in vivo to prevent proteolytic degradation of multiple virulence factors by SpeB. Mol Microbiol 2004, 51:123-134

21. Aziz RK, Kotb M: Rise and persistence of global M1T1 clone of Streptococcus pyogenes. Emerg Infect Dis 2008, 14:1511-1517.

22. Hasegawa T, Okamoto A, Kamimura T, Tatsuno I, Hashikawa SN, Yabutani M, Matsumoto M, Yamada K, Isaka M, Minami M, Ohta M: Detection of invasive protein profile of Streptococcus pyogenes M1 isolates from pharyngitis patients. APMIS 2010, 118:167-178.

23. Tatsuno I, Isaka M, Minami M, Hasegawa T: NADase as a target molecule of in vivo suppression of the toxicity in the invasive $M-1$ group $A$ Streptococcal isolates. BMC Microbiol 2010, 10:144

24. Gryllos I, Grifantini R, Colaprico A, Jiang S, Deforce E, Hakansson A, Telford $J$, Grandi G, Wessels MR: $\mathrm{Mg}^{(2+)}$ signalling defines the group $A$ streptococcal CsrRS (CovRS) regulon. Mol Microbiol 2007, 65:671-683.

25. Trevino J, Perez N, Ramirez-Pena E, Liu Z, Shelburne SA 3rd, Musser JM Sumby P: CovS simultaneously activates and inhibits the CovR-mediated repression of distinct subsets of group A Streptococcus virulence factorencoding genes. Infect Immun 2009, 77:3141-3149.

26. Ferretti JJ, McShan WM, Ajdic D, Savic DJ, Savic G, Lyon K, Primeaux C, Sezate S, Suvorov AN, Kenton S, Lai HS, Lin SP, Qian Y, Jia HG, Najar FZ, Ren Q, Zhu H, Song L, White J, Yuan X, Clifton SW, Roe BA, McLaughlin R: Complete genome sequence of an M1 strain of Streptococcus pyogenes. Proc Natl Acad Sci U S A 2001, 98:4658-4663.

27. Suvorov AN, Ferretti Jj: Physical and genetic chromosomal map of an M type 1 strain of Streptococcus pyogenes. J Bacteriol 1996, 178:5546-5549.

28. Sawai J, Hasegawa T, Kamimura T, Okamoto A, Ohmori D, Nosaka N, Yamada K, Torii K, Ohta M: Growth phase-dependent effect of clindamycin on production of exoproteins by Streptococcus pyogenes. Antimicrob Agents Chemother 2007, 51:461-467.

29. Lukomski S, Hoe NP, Abdi I, Rurangirwa J, Kordari P, Liu M, Dou SJ, Adams GG, Musser JM: Nonpolar inactivation of the hypervariable streptococcal inhibitor of complement gene (sic) in serotype M1 Streptococcus pyogenes significantly decreases mouse mucosal colonization. Infect Immun 2000, 68:535-542

30. Stevens DL, Salmi DB, Mclndoo ER, Bryant AE: Molecular epidemiology of nga and NAD glycohydrolase/ADP-ribosyltransferase activity among Streptococcus pyogenes causing streptococcal toxic shock syndrome. J Infect Dis 2000, 182:1117-1128.

31. Tatsuno I, Sawai J, Okamoto A, Matsumoto M, Minami M, Isaka M, Ohta M, Hasegawa T: Characterization of the NAD-glycohydrolase in streptococcal strains. Microbiology 2007, 153:4253-4260

32. Okada N, Tatsuno I, Hanski E, Caparon M, Sasakawa C: Streptococcus pyogenes protein F promotes invasion of HeLa cells. Microbiology 1998 , 144:3079-3086.

33. Ashbaugh CD, Warren HB, Carey VJ, Wessels MR: Molecular analysis of the role of the group A streptococcal cysteine protease, hyaluronic acid capsule, and $M$ protein in a murine model of human invasive soft-tissue infection. J Clin Invest 1998, 102:550-560.

\section{doi:10.1186/1756-0500-6-126}

Cite this article as: Tatsuno et al:: Partial loss of CovS function in

Streptococcus pyogenes causes severe invasive disease. BMC Research Notes 2013 6:126 